\title{
Hábitos alimentarios e índice de masa corporal en estudiantes de la Universidad del Atlántico, Barranquilla: un estudio descriptivo-transversal
} Eating habits and body mass index in students of
the Universidad del Atlántico, Barranquilla: a cross-
sectional study

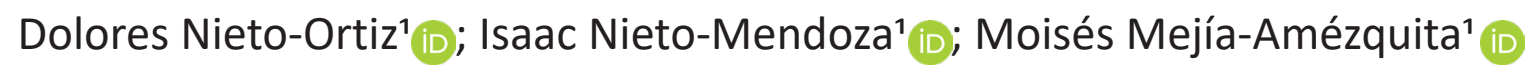

`Universidad del Atlántico. Barranquilla - Atlántico, Colombia; e-mail: doloresniető@hotmail.com; icnieto@mail.uniatlantico.edu.co; moises.elkan@hotmail.com

Cómo citar: Nieto-Ortiz, D.; Nieto-Mendoza, I.; Mejía-Amézquita, M. 2021. Hábitos alimentarios e índice de masa corporal en estudiantes de la Universidad del Atlántico, Barranquilla: un estudio descriptivo-transversal. Rev. Digit. Act. Fis. Deport. 7(1):e1498. http://doi. org/10.31910/rdafd.v7.n1.2021.1498

Artículo de acceso abierto publicado por Revista Digital: Actividad Física y Deporte, bajo una licencia Creative Commons CC BY-NC 4.0

Publicación oficial de la Universidad de Ciencias Aplicadas y Ambientales U.D.C.A, Institución de Educación Superior Acreditada de Alta Calidad por el Ministerio de Educación Nacional.

Recibido: 23 de julio de 2020 Aceptado: 27 de octubre de 2020 Editado por: Álvaro José Gracia Díaz

\section{RESUMEN}

Introducción: La conducta alimentaria del universitario, en el transcurrir de su carrera, se va modificando, a medida que aumentan las responsabilidades académicas y sus implicaciones pueden ser negativas, debido al consumo de alimentos ultraprocesados y altos en grasas, que lo inducen a padecer obesidad o sobrepeso. : Caracterizar los hábitos alimentarios e indicadores de masa corporal de los estudiantes de primer semestre de la Licenciatura en Cultura Física, Recreación y Deportes de la Universidad del Atlántico, en el 2019. Metodología: Estudio descriptivo-transversal, con una muestra de 110 estudiantes de la Universidad del Atlántico, aplicando un cuestionario de hábitos alimentarios y medidas antropométricas (peso y talla), para calcular el Índice de Masa Corporal (IMC). Resultados: Se evidencia un cambio en la conducta alimentaria entre el antes y el después del ingreso a la universidad, como también indicadores de masa corporal, asociados al sobrepeso. Conclusiones: Resulta pertinente concientizar a los universitarios sobre la importancia de la actividad física y la sana alimentación, en aras de fomentar estilos de vida saludable.

Palabras clave: Hábitos alimentarios; Índice de Masa Corporal (IMC); Sobrepeso; Estilos de vida saludable.

\section{ABSTRACT}

Introduction: the university's eating behavior, during the course of his career, is modified as academic responsibilities increase and, its implications can be negative due to the consumption of ultra-processed and high-fat foods that induce him to suffer from obesity or overweight. Course objective: characterize the eating habits and body mass indicators of the first semester students of the Bachelor of Physical 
Culture, Recreation and Sports of the University of the Atlantic in 2019. Methodology: descriptive-crosssectional study with a sample of 110 students from the Universidad del Atlántico, applying a questionnaire of eating habits and anthropometric measurements (weight and height) to calculate the Body Mass Index (BMI). Results: there is a change in eating behavior between before and after university admission, as well as indicators of body mass associated with being overweight. Conclusions: It is pertinent to raise awareness among university students about the importance of physical activity and healthy eating in order to promote healthy lifestyles.

Keywords: Eating habits; Body Mass Index (BMI); Overweight; Healthy lifestyles.

\section{INTRODUCCIÓN}

La obesidad se ha convertido, en la última década, en una epidemia mundial, afectando a individuos de todas las edades, tal y como lo manifiesta el estudio realizado por la Organización Mundial de Salud (OMS, 2020) que, hasta el 2016, "más de 1.900 millones de adultos de 18 o más años, tenían sobrepeso, de los cuales, más de 650 millones eran obesos". Asimismo, la OMS atribuye esta problemática al "aumento en la ingesta de alimentos de alto contenido calórico que son ricos en grasa (...) y el descenso en la actividad física debido a la naturaleza cada vez más sedentaria (...)".

Cabe precisar también, de acuerdo con la investigación realizada por Malo et al. (2017), ha habido un cambio de paradigma, a nivel mundial, con respecto a la obesidad y el sobrepeso, frente a la desnutrición, manifestando que:

"Hace cuarenta años, en el mundo el número de personas con peso bajo era mucho mayor que el de las personas obesas. Esta situación se ha invertido y en la actualidad el número de personas obesas duplica el número de personas con peso bajo. De mantenerse la tendencia, para el año 2030 más de $40 \%$ de la población del planeta tendrá sobrepeso y más de la quinta parte será obesa".

Precisando en investigaciones realizadas con universitarios, en países como México, Chile y España, dan cuenta de la problemática asociada al sobrepeso enlosestudiantes deEducaciónSuperior, relacionando esta situación a los cambios en el estilo de vida, debido al número de horas que permanece sentado en las aulas, gozando también de mayor independencia a la hora de seleccionar los alimentos a consumir, llevando, en la mayoría de ocasiones, a inadecuados hábitos alimentarios (Cutillas et al. 2013; González et al. 2017; Castillo et al. 2016; Aguilar et al. 2010).

Por otra parte, según la Organización para la Cooperación y Desarrollo Económicos (OECD, 2017), a través de su informe Obesity Update, reveló que "en México, Estados Unidos y Reino Unido, se estima que el $47 \%, 39 \%$ y $35 \%$ de la población (respectivamente) serán obesos para el 2030". Estos datos son atribuidos por dicha organización a las siguientes situaciones:

(...) la educación y los bajos niveles socioeconómicos afectan negativamente la salud, la expectativa y la calidad de vida e incrementan las posibilidades de desarrollo de obesidad (...) como también, el consumo de productos y bebidas que, lejos de alimentar, impulsan el sobrepeso y colateralmente el desarrollo de las Enfermedades No Transmisibles (ENT).

Ahora bien, al analizar la situación relacionada con la obesidad y la inseguridad alimentaria en América Latina, la Comisión Económica para América Latina y el Caribe (CEPAL, 2019), afirma que "la prevalencia de la obesidad en adultos en América Latina y el Caribe se ha triplicado desde los niveles de 1975 como consecuencia de un cambio en la alimentación con un mayor consumo de comida rápida y ultraprocesada". Asimismo, el informe de las organizaciones internacionales, como Organización de las Naciones Unidas para la Alimentación y la Agricultura (FAO), Organización Panamericana de la Salud (OPS), el Programa Mundial de Alimentos (WFP), Fondo de las Naciones Unidas para la Infancia (UNICEF), titulado Panorama de la Seguridad Alimentaria y Nutricional en América Latina y el Caribe, reveló que:

(...) el aumento más considerable de la obesidad en adultos en la región se observó en el Caribe, donde el porcentaje se cuadruplicó, pasando de $6 \%$ en 1997 a $25 \%$, un incremento en términos absolutos de 760.000 a 6,6 millones de personas. (...) la publicación destaca que la región está peor que el resto del mundo en la mayoría de los indicadores de malnutrición relacionados con la ingesta excesiva de calorías; el sobrepeso se ha duplicado desde la década de los setenta, y afecta hoy al 59,5\% de los adultos en la región -262 millones de personas-, mientras que a nivel global la tasa es 20 puntos porcentuales menor -39,1\%- (FAO et al. 2019). 
Haciendo énfasis en el contexto colombiano, de acuerdo con la última Encuesta Nacional de la Situación Nutricional (ENSIN), realizada por el Ministerio de Salud y Protección Social, en el 2015, donde se entrevistaron 151.343 colombianos, evidenció que "uno de cada tres jóvenes y adultos tienen sobrepeso $(37,7 \%)$, mientras que uno de cada cinco es obeso (18,7\%). En este sentido, el $56,4 \%$ de la población presenta exceso de peso, lo que significa un incremento de 5,2 puntos porcentualescon respecto al 2010" (Ministerio de Salud y Protección Social, 2015).

También, en el mismo estudio, se identificó que "la obesidad es más frecuente en las mujeres $(22,4 \%)$ que en los hombres $(14,4 \%)$ " (Ministerio de Salud y Protección Social, 2015). Este alto nivel de sobrepeso y obesidad en Colombia, ha estado asociado con la poca práctica de actividad física y la inseguridad alimentaria en el hogar, teniendo presente:

Las responsabilidades de la adultez afectan en muchos casos la alimentación, dedicándole poco tiempo, consumiendo más alimentos procesados y menos comidas preparadas en casa. La oferta de alimentos procesados, frituras y dulces, así como una vida sedentaria, propician el desarrollo de obesidad muchas veces asociada a deficiencias nutricionales (Ministerio de Salud y Protección Social, 2015).

Con relación a la problemática en las universidades colombianas, investigaciones han develado la prevalencia de sobrepeso y obesidad, epidemias asociadas con los hábitos alimentarios poco saludables y la baja práctica de actividad física. Esto se relaciona, a su vez, con el estrés al que están expuestos estudiantes en el desarrollo de sus jornadas académicas, que conllevan al consumo de alimentos de fácil y rápida preparación y el sedentarismo (Fernández et al. 2018; Cardozo et al. 2016; Becerra et al. 2016; Canova, 2017; Álvarez et al. 2014).

Entre tanto, al analizar la situación actual del departamento del Atlántico, de acuerdo con el informe del Ministerio de Salud y Protección Social, a través de la ENSIN, del 2015, 56 de cada 100 jóvenes y adultos entre 18 y 64 años, "tienen exceso de peso (...) a su vez, tanto en el departamento del Atlántico como Barranquilla y su área metropolitana, 60 adultos de cada 100 tiene exceso de peso" (Ministerio de Salud y Protección Social, 2017).
A nivel universitario, en Barranquilla, investigaciones reconocen la tendencia de los estudiantes hacia la obesidad y, especialmente, hacia el sobrepeso, asociado con el estilo de vida de los universitarios y la inseguridad alimentaria del hogar. Además, muchos estudiantes no se alimentan adecuadamente, recurriendo al reemplazo de alimentos esenciales con comidas rápidas y procesados, como también la poca práctica de actividad física (Kuzmar et al. 2018; Miranda et al. 2015; Suárez et al. 2019).

Finalmente, en lo que respecta a la Universidad del Atlántico, la investigación realizada por Nieto \& Torrenegra (2019), dio cuenta de los problemas, que se aprecian en universitarios con relación al exceso de peso, a través de estudio de 207 universitarios, en el 2018, donde el sobrepeso y la inactividad física fueron constantes entre los resultados.

Es así, como el interés de esta investigación precisa en abordar a los estudiantes de primer semestre en la Licenciatura en Cultura Física y Recreación y Deportes, teniendo presente, que se hace notorio, en muchos de ellos, exceso de peso y algunos poseen hábitos alimentarios poco saludables y otras conductas distantes, de estilos de vida saludables.

\section{MATERIALES Y MÉTODOS}

Estudio descriptivo-transversal realizado con estudiantes de primer semestre del programa Licenciatura en Cultura Física, Recreación y Deportes de la Universidad del Atlántico, en el 2019, estando la muestra conformada por 110 universitarios.

\section{Procedimientos e instrumentos.}

Hábitos alimentarios: Para el análisis de la clase de alimentosconsumidosporlosuniversitarios, seaplicóel instrumento utilizado en la investigación realizada por Barrera et al. (2008), con universitarios colombianos, teniendo en cuenta las Guías Alimentarias del Plan Nacional de Alimentación y Nutrición del Instituto Colombiano de Bienestar Familiar (ICBF).

Índice de Masa Corporal (IMC): Para calcular el IMC, se tuvieron en cuenta los baremos recomendados por la Organización Mundial de la Salud (OMS), para población adulta y utilizados por Nieto \& Torrenegra (2019), en estudiantes de la Universidad 
del Atlántico, siendo estos "bajo peso $\left(<18,5 \mathrm{~kg} / \mathrm{m}^{2}\right)$, normal $\left(18,5 \mathrm{~kg} / \mathrm{m}^{2}\right.$ a $\left.24,9 \mathrm{~kg} / \mathrm{m}^{2}\right)$, sobrepeso $(>25,0 \mathrm{~kg} /$ $\mathrm{m}^{2}$ a $\left.29,9 \mathrm{~kg} / \mathrm{m}^{2}\right)$ y obesidad $\left(>30,0 \mathrm{~kg} / \mathrm{m}^{2}\right)^{\prime \prime}$.

Para tomar el peso, se utilizó una balanza electrónica de pie marca Lifesource MD $^{\circledR}$, Modelo: CW184015, sensibilidad $5 \mathrm{~g}$ y capacidad de $250 \mathrm{~kg}$; se solicitó a los estudiantes despojarse de sus zapatos, con la intención de hacer la medición precisa. Para la toma de la estatura, se utilizó un tallímetro portátil marca Seca bodymeter $208^{\circledR}$ sensibilidad de $5 \mathrm{~mm}$. El tallímetro fue fijado en una pared.

Consideraciones éticas. Entre las consideraciones legales, se tuvo en cuenta lo expuesto en la Resolución 8430 de 1993, con respecto al consentimiento informado, como se expone en el Artículo 6, como también los criterios para la clasificación de la investigación, como sin riesgo. Análisis estadístico. El análisis estadístico, se realizó a través de la herramienta de Microsoft Office Excel, mostrando los resultados a través de tablas y gráficas.

\section{RESULTADOS}

Caracterización sociodemográfica. De acuerdo con las características demográficas presentadas en la tabla 1, el rango de edad preponderante fue de 23 a 27 años (78,2\%). Entre tanto, la edad promedio fue de $24.3 \pm 5.7$, con un mínimo de 18 y un máximo de 30 años. La mayoría de estudiantes son de género masculino $(86,4 \%)$, siendo el estrato socioeconómico 1 de mayor frecuencia, con un 60,9\%. Finalmente, en cuanto a con quién conviven actualmente, gran parte de la muestra afirma convivir con personas sin vínculos afectivos familiares $(66,4 \%)$.

Tabla 1. Características demográficas de la muestra estudiada.

\begin{tabular}{|c|c|c|}
\hline Características & $\mathbf{n}$ & $\%$ \\
\hline \multicolumn{3}{|l|}{ Edad en años: } \\
\hline $18-22$ & 86 & $78,2 \%$ \\
\hline $23-27$ & 19 & $17,3 \%$ \\
\hline $28-32$ & 5 & $4,5 \%$ \\
\hline \multicolumn{3}{|l|}{ Género: } \\
\hline Masculino: & 95 & $86,4 \%$ \\
\hline Femenino: & 15 & $13,6 \%$ \\
\hline \multicolumn{3}{|l|}{ Estrato socioeconómico: } \\
\hline 1 & 67 & $60,9 \%$ \\
\hline 2 & 32 & $29,1 \%$ \\
\hline 3 & 8 & $7,3 \%$ \\
\hline 4 & 3 & $2,7 \%$ \\
\hline \multicolumn{3}{|l|}{ Actualmente convive: } \\
\hline Solo (a) & 15 & $13,6 \%$ \\
\hline Con la familia nuclear: & 22 & $20,0 \%$ \\
\hline Con personas sin vínculos familiares: & 73 & $66,4 \%$ \\
\hline
\end{tabular}

Evaluación de hábitos alimentarios. Tras los resultados presentados en la tabla 2, es preciso aseverar que, antes de ingresar a la universidad, la frecuencia de estudiantes que almorzaban fue mayor a la frecuencia que se aprecia en cuanto a desayuno y comida, representada en un $100 \%$ de los universitarios, que aseveran acostumbraban almorzar. Asimismo, el número de veces por semana en que desayunaban, almorzaban o comían representó mayor porcentaje en el almuerzo, que era más de tres días, a diferencia de las comidas, donde la mayoría de universitarios aseveró acostumbrar hacerlo 3 o menos días. 
Tabla 2. Distribución de desayuno, almuerzo y comida de los universitarios

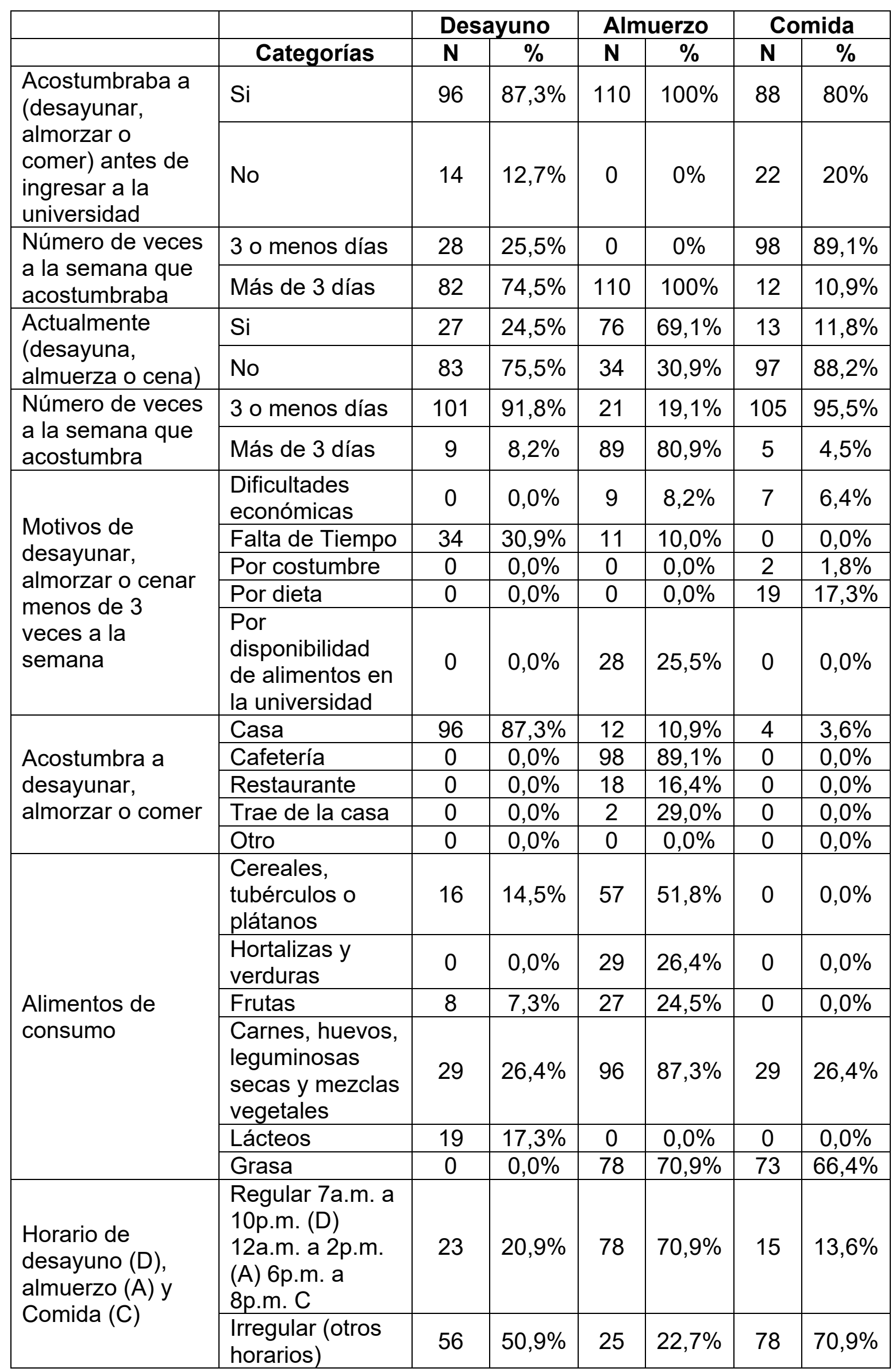


Ahora bien, en cuanto al ingreso a la universidad, se evidencia una disminución considerable en el consumo de alimentos en el desayuno (75\%) y en el almuerzo $(69,1 \%)$; también, se hace palmario un porcentaje llamativo con respecto al número de veces a la semana que acostumbra a desayunar, afirmando los universitarios hacerlo 3 o menos días (91,8\%).

Entre tanto, entre los motivos por los cuales el universitario actualmente desayuna, almuerza o come 3 días a la semana o menos, se destacan, la falta de tiempo, especialmente en los desayunos, y por la disponibilidad de alimentos en la universidad, en especial, en el almuerzo. Con relación al lugar donde los universitarios acostumbran a desayunar, almorzar o comer, la mayoría selecciona la casa, como el espacio donde consume alimentos matutinos (desayuno), la cafetería de la universidad, el restaurante y el consumo de alimentos traídos de la casa en los almuerzos.
Con respecto a los alimentos de consumo, en el desayuno, se aprecia un consumo de cereales, tubérculos y plátanos, como también huevos y lácteos; en el almuerzo hay un mayor consumo de tubérculos, productos cárnicos y frutas y alimentos con alto contenido en grasas $y$, por último, con respecto a las comidas, se aprecia un consumo de carnes y de alimentos altos en grasas.

Finalmente, en cuanto a los horarios de desayuno almuerzo y comida, se hace notorio un porcentaje considerable en los tiempos de consumo de las comidas, como también en los desayunos. Por su parte, en cuanto al almuerzo, gran parte de los universitarios manifiestan hacerlo en el horario regular, de $7 \mathrm{am}$ a $10 \mathrm{pm}$ (D), $12 \mathrm{am}$ a $2 \mathrm{pm}(\mathrm{A}), 6 \mathrm{pm}$ a $8 p m(C)$.

Resultados Índice de Masa Corporal (IMC). El Índice de Masa Corporal (IMC) fue calculado, teniendo en cuenta el peso y la talla, obteniendo los resultados presentados en la figura 1.

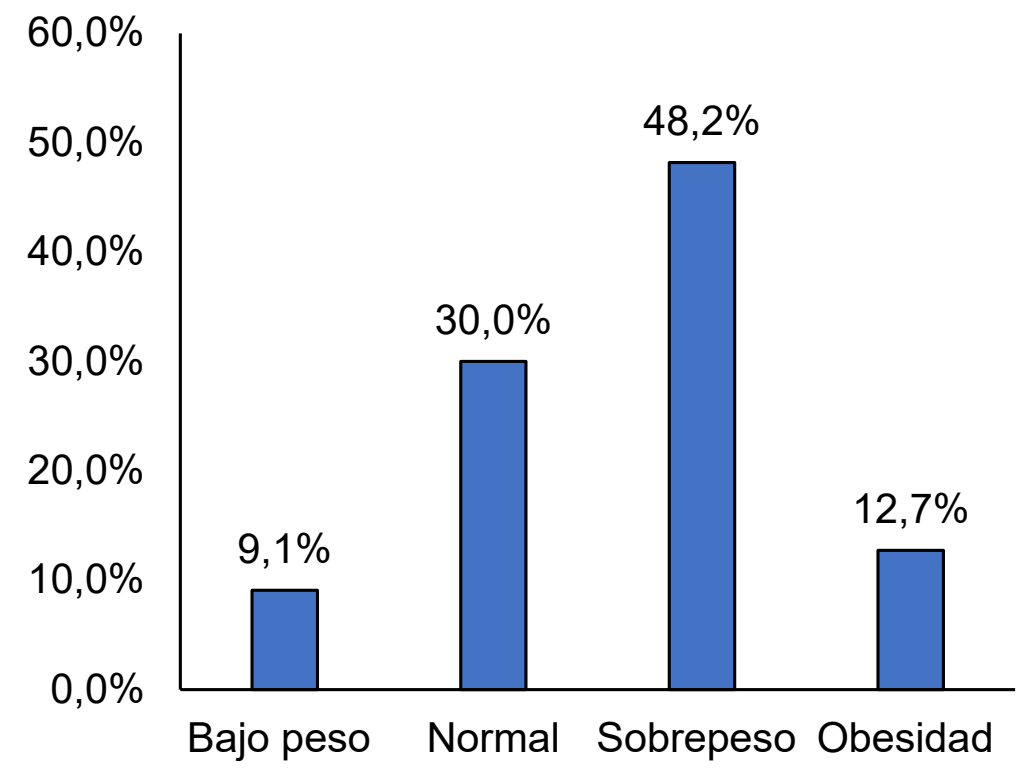

Figura 1. Indicadores de Masa Corporal de universitarios. 
Al calcular los niveles de masa corporal asociados con el bajo peso es notoria una minoría de los universitarios que se encuentran en este indicador, $<18,5 \mathrm{~kg} / \mathrm{m}^{2}$, representada en el $9,1 \%$ y con una medida promedio de $16,6 \pm 7,2$. Por su parte, la obesidad, $>30,0 \mathrm{~kg} / \mathrm{m} 2$, fue el segundo indicador que reportó minoría de frecuencia, con un $12,7 \%$, con una medida promedio de $34,7 \pm 9,2$.

Por su parte, en los indicadores que reportaron mayor frecuencia de los estudiantes, el índice de masa corporal normal, $18,5 \mathrm{~kg} / \mathrm{m}^{2}$ a $24,9 \mathrm{~kg} / \mathrm{m}^{2}$, reportó un $30 \%$, con una medida promedio de $22,5 \pm 6,2$; por último, el indicador con mayor porcentaje de universitarios fue el sobrepeso, $>25,0 \mathrm{~kg} / \mathrm{m} 2$ a $29,9 \mathrm{~kg} /$ $\mathrm{m}^{2}$, con un $48,2 \%$ y una medida promedio de $26,3 \pm 4,3$.

\section{DISCUSIÓN}

Tras los resultados obtenidos con relación a los hábitos alimentarios de los universitarios de la Licenciatura en Cultura Física, Recreación y Deporte es posible evidenciar un cambio en relación con sus conductas alimentarias al ingresar a la universidad, especialmente, en cuanto al consumo de desayuno y el número de veces en que se consume el almuerzo, debido a que, al no tener responsabilidades educativas, consume el almuerzo más de 3 veces al día. Este resultado concuerda con la investigación realizada por Becerra et al. (2016), en la que se hicieron palmarios cambios asociados con los hábitos alimentarios de universitarios, debido a medida que avanzaban en su carrera. Según Ruíz et al. (2014), el universitario puede ver condicionada su conducta alimentaria, debido a largas jornadas académicas.

Por otra parte, en cuanto a los motivos por los que los universitarios desayunan, almuerzan o cenan menos de 3 veces a la semana, esta investigación identificó la falta de tiempo y la poca disponibilidad de alimentos en la universidad, relacionándose con los resultados obtenidos por Galeano et al. (2018), donde los estudiantes de una universidad, de Barranquilla, aseveraban tener hábitos alimentarios poco saludables, debido a que las responsabilidades académicas, muchas veces, se extendían a las horas de almuerzo, consumiendo alimentos ultra-procesados comercializados dentro de la universidad, por ser de fácil y rápido acceso; esto se asocia, a su vez, con el estrés al que están expuestos los estudiantes en el desarrollo de sus jornadas académicas, que conllevan al consumo de alimentos de fácil y rápida preparación (Fernández et al. 2018; Cardozo et al. 2016; Becerra et al. 2016; Canova, 2017; Álvarez et al. 2014).

Diversas investigaciones manifiestan que la vida universitaria trae ciertas implicaciones relacionadas con la autonomía del joven, quien ingresa al contexto estudiantil, debido a que posee la libertad para tomar decisiones referentes a su estilo de vida (Nieto \& Torrenegra, 2019; Benítez, 2016; Canova, 2017; Cardozo et al. 2016). Es por ello, que el universitario toma la decisión de consumir alimentos que se comercializan a los alrededores de la universidad, desprendiéndose de las comidas hechas en casa, accediendo alimentos con alto contenido en grasas y pocas propiedades energéticas.

En lo que concierne al tipo de alimentos consumidos por los universitarios, llama la atención el bajo consumo de frutas y verduras, como también la ingesta alimentaria basada en alimentos con alto contenido de grasas, tanto en el almuerzo como en la comida, conllevando, entonces, a la inclusión de su plan alimentario el consumo de alimentos de fácil y rápida preparación, como comidas rápidas, fritos, bebidas azucaradas, dulces y ultra-procesados, que favorecen el desarrollo de la obesidad y enfermedades no transmisibles (OMS, 2020; Oliva \& Fragoso, 2013); no obstante, a pesar que en la investigación los indicadores de masa corporal asociados a la obesidad no fueron representativos, se hace palmaria una tendencia al sobrepeso que representa, según la OMS (2016), un factor de riesgo para padecer enfermedades crónicas o transmisibles, como las cardiopatías, los accidentes cardiovasculares, la diabetes y el cáncer.

Cabe precisar también, que el consumo de alimentos a destiempo tiene un impacto en la salud del individuo, conllevando al consumo excesivo de alimentos (Chacón-Cuberos et al. 2016) y representa un factor de riesgo para presentar sobrepeso (Nieto \& Torrenegra, 2019; Lara \& Nieto, 2019). Esta investigación dio cuenta, que los universitarios tienen horarios irregulares para el consumo de desayuno y comida, lo cual hace que estén expuestos a padecer enfermedades no transmisibles, debido a conductas alimentarias insanas. 


\section{CONCLUSIONES}

Resulta fundamental que los universitarios sean inducidos a la práctica de actividad física y al mejoramiento de sus hábitos alimentarios, con el fin de reducir los factores de riesgo para el padecimiento de enfermedades crónicas no transmisibles, buscando, además, consolidar la idea de un contexto universitario saludable. Por tanto, la creación de programas universitarios que busquen la participación activa de toda la comunidad es determinante para que docentes, estudiantes, administrativos y otros, que participen de actividades informativas, donde se les brinden pautas nutricionales y se hagan, además, valoraciones antropométricas, para conocer las necesidades de la comunidad estudiantil de la Universidad del Atlántico, a nivel nutricional.

Conflicto de intereses. Los autores declaramos la no existencia de conflictos de intereses de ninguna índole, ni real ni potencial de los resultados presentados.

\section{REFERENCIAS}

1. AGUILAR, A.; PÉREZ, D.; RODRÍGUEZ, L.; HERNÁNDEZ, S.; JIMÉNEZ, F.; RODRÍGUEZ, R. 2010. Prevalencia estacionaria de sobrepeso y obesidad en universitarios del sur de Veracruz, México. Medicina Universitaria. 12(46):24-28.

2. ÁLVAREZ, L.; SALAZAR, A.; DÍAZ, Y. 2014. Relación entre las emisiones de dióxido de carbono equivalentes por consumo de carne y el índice de masa corporal, el peso y el consumo diario de kilocalorías en estudiantes de a Universidad del Magdalena, Santa Marta, 2013. Médicas UIS. 27(3):47-55.

3. BARRERA, J.; BICENTY, A.; GERENA, J.; IBAÑEZ, E.; MARTÍNEZ, J.; THOMAS, Y. 2008. Cambios de hábitos alimentarios de los estudiantes de odontología de la Fundación Universitaria San Martín de Bogotá, Colombia. Nova-Publicación Científica en Ciencias Biomédicas. 6(9):101112.

https://doi.org/10.22490/24629448.393

4. BECERRA, C.; PINZÓN, G.; VARGAS, M.; MARTÍNEZ, E.; CALLEJAS, E. 2016. Cambios en el estado nutricional y hábitos alimentarios deestudiantes universitarios, Bogotá D.C. 2013. Revista de la Facultad de Medicina. 64(2):249-56.

http://dx.doi.org/10.15446/revfacmed.v64n2.50722
5. BENÍTEZ, A. 2016. Hábitos alimentarios de riesgo en la población universitaria extremeña. Tesis Doctoral. Universidad de Extremadura (Extremadura, España).

6. CANOVA, C. 2017. Estilo de vida de estudiantes universitarios de enfermería de Santa Marta, Colombia. Revista Colombiana de Enfermería. 14(12):23-32. http://dx.doi.org/10.18270/rce.v14i.2025

7. CARDOZO, L.; CUERVO, Y.; MURCIA, J. 2016. Porcentaje de grasa corporal y prevalencia de sobrepeso - obesidad en estudiantes universitarios de rendimiento deportivo de Bogotá, Colombia. Nutrición Clínica y Dietética Hospitalaria. 36(3):68-75. https://doi.org/10.12873/363cardozo

8. CASTILLO, I.; SOLANO, S.; SEPÚlVEDA, A. 2016. Programa de prevención alteraciones alimentarias y obesidad en estudiantes universitarios mexicanos. Psicologia Conductual. 24(1):5-28.

9. CEPAL. 2019. La obesidad se triplica en América Latina por un mayor consumo de ultraprocesados y comida rápida. Noticias ONU. Naciones Unidas.

10. CHACÓN-CUBEROS, R.; CASTRO-SÁNCHEZ, M.; MUROS-MOLINA, J.J.; ESPEJO-GARCÉS, T.; ZURITA-ORTEGA, F.; LINARES-MANRIQUE, M. 2016. Adhesión a la dieta mediterránea en estudiantes universitarios y su relación con los hábitos de ocio digital. Nutrición Hospitalaria. 33(2):405-410

11. CUTILLAS, A.; HERRERO, E.; DE SAN EUSTAQUIO, A.; ZAMORA, S.; PÉREZ, S. 2013. Prevalencia de peso insuficiente, sobrepeso y obesidad, ingesta de energía y perfil calórico de la dieta de estudiantes universitarios de la Comunidad Autónoma de la Región de Murcia (España). Nutrición Hospitalaria. 28(3):683-689. https://doi.org/10.3305/nh.2013.28.3.6443

12. FAO; OPS; WFP; UNICEF. 2019. Panorama de la seguridad alimentaria y nutrición en América Latina y el Caribe. Naciones Unidas (Santiago).

13. FERNÁNDEZ, J.; STIC, H.; SANTAMARÍA, O.; RAMOS, S. 2018. Relación entre consumo de oxígeno, porcentaje de grasa e índice de masa corporal en universitarios. Hacia la Promoción de la Salud. 23(2):79. 
14. GALEANO, L.; PINILLOS, Y.; HERAZO, Y.; GONZÁLEZ, J.; LÓPEZ, A. 2018. Factores de riesgo para enfermedad crónica no transmisible en jóvenes universitarios de un programa de las ciencias de la salud de Barranquilla. Revista Latinoamericana de Hipertensión. 13(4):380-385.

15. GONZÁLEZ, L.; CARREÑO, C.; ESTRADA, A.; MONSALVE, J.; ÁlVAREZ, L. 2017. Exceso de peso corporal en estudiantes universitarios según variables sociodemográficas y estilo de vida. Revista Chilena de Nutrición. 44(3):251- 261. http://dx.doi.org/10.4067/s071775182017000300251

16. KUZMAR, I.; CONSUEGRA, J.; CORTÉS, E.; RIZO, M.; ALMANZA, C.; ANTONIO, K.; DE LA HOZ, M.; GONZÁLEZ, M.; GUERRERO, D.; MORALES, K.; MORENO, S.; PEÑATE, K.; PIANETA, K.; RADA, L.; RAMIREZ, O.; REDONDO, D.; SOLANO, S.; SUÁREZ, L.; VARGAS, J.; BERMÚDEZ, V.; VILLA, A. 2018. Hábitos y estado nutricional relacionados con las diferentes carreras universitarias. Revista Latinoamericana de Hipertensión. 13(4):361-367.

https://doi.org/10.6084/m9.figshare.5830659

17. LARA, B.; NIETO, I. 2019. Percepción de la imagen corporal en mujeres de 25 años del municipio de ponedera (Atlántico). Biociencias. 14(4):113-127. https://doi.org/10.18041/2390-0512/ biociencias.2.6025

18. MALO, M.; CASTILLO, N.; PAJITA, D. 2017. La obesidad en el mundo. Anales de la Facultad de Medicina. 78(2):173-178.

http://dx.doi.org/10.15381/anales.v78i2.13213

19. MINISTERIO DE SALUD Y PROTECCIÓN SOCIAL. 1993. Resolución 8430 de 1993. Por la cual se establecen las normas científicas, técnicas y administrativas para la investigación en salud.

20. MINISTERIO DE SALUD Y PROTECCIÓN SOCIAL. 2015. Encuesta Nacional de la Situación Nutricional. Nota de Política (Bogotá).

21. MINISTERIO DE SALUD Y PROTECCIÓN SOCIAL. 2017. Barranquilla recibió resultados de Encuesta Nacional de Situación Nutricional de Colombia (ENSIN) 2015. Boletín de prensa No 177 de 2017.
22. MIRANDA, Y.; RODRÍGUEZ, M.; MOLINARES, F.; PEÑA, A.; GARCÍA, F. 2015. Niveles de actividad física en los estudiantes de la facultad de ciencias de la salud de la Universidad Simón Bolívar de Barranquilla. Revista Salud en Movimiento. 7(1):22-28.

23. NIETO, D.; TORRENEGRA, S. 2019. Relación entre el índice de masa corporal y actividad física en universitarios de la ciudad de Barranquilla en el semestre 2018-2, Universidad del Atlántico. Biociencias. 14(1):151-164. https://doi.org/10.18041/2390-0512/ biociencias.1.5441

24. OECD. 2017. Obesity Update. OECD Better Polices for Better Lives. 13p.

25. OLIVA CHÁVEZ, O.; FRAGOSO, S. 2013. Consumo de comida rápida y obesidad, el poder de la buena alimentación en la salud. Revista Iberoamericana para la Investigación y el Desarrollo Educativo. 4(7):176-199.

26. OMS. 2016. ¿Qué es la malnutrición? Organización Mundial de la Salud.

27. OMS. 2020. Obesidad y sobrepeso. Organización Mundial de la Salud. Disponible desde Internet en: https://www.who.int/es/news-room/ fact-sheets/detail/obesity-and-overweight

28. RUÍZ, E.; DEL POZO, S.; VALERO, T.; ÁVILA, J.; VARELA, G. 2014. Estudio de hábitos alimentarios y estilos de vida de los universitarios españoles. Fundación Española de la Nutrición.

29. SUÁREZ, O.; VILLAREAL, C.; PARODY, A.; RODRÍGUEZ, A.; REBOLLEDO, R. 2019. Prevalencia de hipertensión arterial y de sus factores de riesgo en estudiantes universitarios de Barranquilla, Colombia. Revista de la Facultad de Ciencias de la Salud de la Universidad del Cauca. 21(2):16-23. 